\title{
The problem of physical coordinates in predictive Hamiltonian systems
}

\author{
V. Iranzo, J. Llosa, F. Marqués, and A. Molina \\ Departament de Física Teòrica, Universitat de Barcelona, Barcelona-28, Spain
}

(Received 1 December 1981; accepted for publication 11 August 1982)

\begin{abstract}
In the Hamiltonian formulation of predictive relativistic systems, the canonical coordinates cannot be the physical positions. The relation between them is given by the individuality differential equations. However, due to the arbitrariness in the choice of Cauchy data, there is a wide family of solutions for these equations. In general, those solutions do not satisfy the condition of constancy of velocities' moduli, and therefore we have to reparametrize the world lines into the proper time. We derive here a condition on the Cauchy data for the individuality equations which ensures the constancy of the velocities' moduli and makes the reparametrization unnecessary.
\end{abstract}

PACS numbers: 11.80. $-\mathrm{m}, 02.30 . \mathrm{Jr}, 11.30 . \mathrm{Cp}$

\section{INTRODUCTION}

The description of the relativistic interaction at a distance between particles has been a difficult task both from the mathematical and the conceptual viewpoints. There are some different ways to face these problems in the literature. $^{1-4}$

In this paper we consider two versions of an action-at-adistance theory called predictive relativistic mechanics (PRM). The main hypothesis of this theory are: (i) Poincaré invariance and (ii) the dynamics of the interacting particles is given by a second-order differential system and, therefore, the motion is completely determined by the initial positions and velocities. The versions of the PRM which we consider here are the so called "multitime formalism"s and " $a$ priori Hamiltonian formulation," 6 respectively.

In the multitime formalism, Hamiltonian descriptions have been derived by Bel and co-workers ${ }^{7}$ for the electromagnetic interaction and for the scalar and vector short range ones. However, this method leads inevitably from the beginning to power expansions in some constant and, although there are iterative algorithms to obtain all interesting functions order by order, their convergence has not been proved yet. Also, it is hard to go beyond order two.

In the a priori Hamiltonian framework ${ }^{6}$ it is possible to consider a wide class of models which do not need perturbative methods, at least in the first steps. However, this formalism is objected to in that it does not allow us to describe physical interactions like those mentioned above. Anyhow, the a priori Hamiltonian formalism will provide exact simple models of interacting relativistic particles whose study will permit us a better understanding of quantization.

Both frameworks can be related to one another by integrating the so-called position equations-see Sec. II. As we shall see later, a Poincaré-invariant predictive system (multitime formalism) can be associated with each " $a$ priori" $\mathrm{Ha}$ miltonian one plus a good set of Cauchy data for the position equations. However, this correspondence usually implies reparametrization of the world lines of each particle into its individual proper time. This is studied in detail in Secs. II and III.

Because of the necessity of reparametrizing the world lines, the associated Poincaré-invariant predictive system does not coincide with the starting Hamiltonian one, but they are proportional, as we shall see in Sec. III. Therefore, the former does not leave the symplectic form invariant whereas the Hamiltonian system does.

As we have mentioned above, an "a priori" Hamiltonian system is in correspondence with a wide class of Poincaré-invariant predictive ones, depending on the prescribed Cauchy data. In Secs. IV and V we study how the Cauchy data can be restricted in order to ensure the coincidence between the "a priori" Hamiltonian system and the associated Poincaré-invariant predictive one.

\section{PREDICTIVE HAMILTONIAN SYSTEMS}

In predictive relativistic mechanics the basic equations of motion consist of $N$ coupled second-order differential systems 8

$$
\begin{aligned}
& \frac{\partial x_{a}^{\mu}}{\partial \sigma_{b}}=\delta_{a b} \pi_{a}^{\mu}, \quad \frac{\partial \pi_{a}^{\mu}}{\partial \sigma_{b}}=\delta_{a b} \theta_{a}^{\mu}(x, \pi), \\
& a, b, c, \cdots=1, \ldots, N, \quad \mu, v, \cdots=0,1,2,3 .
\end{aligned}
$$

Each one defines a vector field on the phase space $T M^{4 N}$

$$
\mathbf{H}_{a}=\pi_{a}^{\mu} \cdot \frac{\partial}{\partial x^{a \mu}}+\theta_{a}^{\mu}(x, \pi) \cdot \frac{\partial}{\partial \pi^{a \mu}}, \quad a=1, \ldots, N .
$$

The integrability conditions of (2.1) read

$$
\left[\mathbf{H}_{a}, \mathbf{H}_{a^{\prime}}\right]=0, \quad \forall a^{\prime} \neq a,
$$

meaning that if, starting from a point $P$ in the phase space, some among the particles are transported along their respective world lines to a new point $Q \in T M^{4 N}$, the same integral of $(2.1)$ is obtained irrespectively that you take either $P$ or $Q$ as initial conditions.

It is easy to proof the following ${ }^{9,10}$ :

Theorem 2.1: Let $\mathbf{H}_{1}, \ldots, \mathbf{H}_{N}$ be vector fields of the type (2.2) such that $\left[\mathbf{H}_{a}, \mathbf{H}_{a^{\prime}}\right]=0, \forall a^{\prime} \neq a$. Then, there exists an open neighborhood $\mathscr{U}$ of $\{0\} \times T M^{4 N} \subset \mathbb{R}^{N} \times T M^{4 N}$ and a continuously differentiable mapping

$$
\begin{aligned}
& \varphi: \mathscr{U} \rightarrow T M^{4 N}, \\
& \left(\sigma_{1}, \ldots, \sigma_{N} ; P\right) \rightarrow\left(\varphi_{a}^{\mu}\left(\sigma_{a}, x_{b}^{v}, \pi_{c}^{\rho}\right), \dot{\varphi}_{a}^{\mu}\left(\sigma_{a}, x_{b}^{v}, \pi_{c}^{\rho}\right)\right), \\
& P=\left(x_{b}^{v}, \pi_{c}^{\rho}\right),
\end{aligned}
$$

such that: 
(i) $\varphi(0, P)=P, \quad \forall P \in T M^{4 N}, \quad 0=(0, \ldots, 0) \in \mathbb{R}^{N}$,

(ii) $\left(\sigma^{2}, P\right) \in \mathscr{U}, \quad\left(\sigma^{1}, \varphi\left(\sigma^{2}, P\right)\right) \in \mathscr{U}, \quad\left(\sigma^{1}+\sigma^{2}, P\right) \in \mathscr{U}$

$\Rightarrow \varphi\left(\sigma^{1}, \varphi\left(\sigma^{2}, P\right)\right)=\varphi\left(\sigma^{1}+\sigma^{2}, P\right)$;

(iii) for any function $f$ on $T M^{4 N}$,

$$
\left[\frac{\partial}{\partial \sigma_{a}} f\left(\varphi\left(\sigma_{1}, \ldots, \sigma_{N} ; P\right)\right)\right]_{\sigma_{i}=0}=\left(\mathbf{H}_{a} f\right)_{P}
$$

(this means that $\mathbf{H}_{a}$ is the tangent vector to the trajectory of the particle $a$ in the phase space);

(iv) uniqueness.

A very important feature due to the Kronecker $\delta$ 's appearing in (2.1) is that $\varphi_{a}^{\mu}$ depends only on the parameter $\sigma_{a}$. This property is called the "individuality condition."

Theorem 2.2: Let $Q$ be a neighborhood of $\{0\} \times T M^{4 N} \subset \mathbb{R}^{N} \times T M^{4 N}$ and let $\varphi$ be a continuously differentiable mapping:

$$
\begin{aligned}
& \varphi: \mathscr{U} \rightarrow T M^{4 N} \\
& \left(\sigma_{1}, \ldots, \sigma_{N} ; P\right) \rightarrow\left(\varphi_{a}\left(\sigma_{a}, P\right), \dot{\varphi}_{a}\left(\sigma_{a}, P\right)\right)
\end{aligned}
$$

with the properties (i) and (ii).

Then the vector fields $\left\{\mathbf{H}_{a}\right\}_{a=1 \ldots N}$ defined by (2.6) are of the type (2.2) and fulfill the conditions (2.3).

The Poincaré invariance of the differential system (2.1) is assured iff $\theta_{a}^{\mu}$ are translation-invariant 4-vectors. Then

$$
\left[\mathbf{P}_{\mu}, \mathbf{H}_{a}\right]=0, \quad\left[\mathbf{J}_{\mu \nu}, \mathbf{H}_{a}\right]=0,
$$

where $\mathbf{P}_{\mu}$ and $\mathbf{J}_{\mu \nu}$ are the generators of the Poincaré group.

Equations (2.3) and (2.7) exhibit that $\mathbf{P}_{\mu}, \mathbf{J}_{\mu \nu}$, and $\mathbf{H}_{a}$ span a realization of an abelian extension of the Poincaré algebra. ${ }^{7}$ The associated transformation group $\mathscr{P} \otimes \mathscr{A}_{N}$ is the direct product of the Poincare group and the abelian one $\mathscr{A}_{N} \simeq \mathbb{R}^{N}$.

Hereafter will be called a Poincaré-invariant abelian system (PIAS) any family of differential systems $\left\{\mathbf{H}_{a}\right.$, $a=1, \ldots, N\}$ of the type (2.2) fulfilling (2.3) and (2.7).

In predictive relativistic mechanics it is also required that $\pi_{a}^{2}$ be an integral of motion for any system $\left\{\mathbf{H}_{b}, b=1, \ldots, N\right\}$. This means that the parameter $\sigma_{a}$ is the proper time of particle $a$, apart from a multiplicative constant. This implies the following relation:

$$
\pi_{a \mu} \cdot \theta_{a}^{\mu}(x, \pi)=0 \text {. }
$$

A PIAS satisfying the conditions (2.8) will be called hereafter a Poincaré-invariant predictive system (PIPS).

A Hamiltonian formalism for the system (2.1) will be determined by picking up a simplectic $\Omega$ form on $T M^{4 N}$ invariant under $\mathscr{P} \otimes \mathscr{A}_{N}$.

As is well known, a function $f$ can be associated to each field $\Lambda$, which leaves $\Omega$ invariant. This function is defined in $T M^{4 N}$, at least locally, by

$$
d f=i_{\Lambda} \Omega
$$

and it is unique, apart from an arbitrary additive constant [in Eq. (2.8) $i$ means the interior product $\left.{ }^{9}\right]$.

In particular, the functions associated with $\mathbf{P}_{\mu}, \mathbf{J}_{\mu \nu}$, and $\mathbf{H}_{a}$ are, respectively, the linear momentum, the angular momentum, and the Hamiltonians.

It is important to realize that in all cases of interest: $\Omega \neq d x_{a}^{\mu} \wedge d \pi_{\mu}^{a}$ in order to circumvent the no-interaction theorem. $^{3}$

The preceding are the main features of the natural approach to obtain a Hamiltonian description [as a first step to quantization of a classical system like (2.1)]. A very important fact of this approach is that it usually involves perturbative methods, as it can be seen in the cases of electromagnetic and of short range scalar and vector interactions. ${ }^{7,11}$

Another approach to predictive relativistic mechanics is given by the so-called "a priori Hamiltonian predictive systems." It consists in giving $N$ scalar functions $H_{a}(q, p)$ on the phase space $T M^{4 N}$ endowed with a symplectic form:

$$
\Omega=d q_{a}^{\mu} \wedge d p_{\mu}^{a}
$$

where, in order to assure the Poincaré invariance, the canonical coordinates $q_{a}^{\mu}$ behave like $x_{a}^{\mu}$ under Poincaré transformations and the momenta $p_{\mu}^{a}$ are translation invariant 4vectors. This implies

$$
\begin{aligned}
& \mathbf{P}_{\mu}=\epsilon_{a} \frac{\partial}{\partial q_{a}^{\mu}} \\
& \mathbf{J}_{\mu v}=q_{a \mu} \cdot \frac{\partial}{\partial q_{a}^{v}}-q_{a v} \cdot \frac{\partial}{\partial q_{a}^{\mu}}+p_{\mu}^{a} \cdot \frac{\partial}{\partial p^{a v}}-p_{v}^{a} \cdot \frac{\partial}{\partial p^{a \mu}}
\end{aligned}
$$

and the corresponding functions:

$$
P_{\mu}=\epsilon_{a} p_{\mu}^{a}, \quad J_{\mu v}=q_{a \mu} \cdot p_{v}^{a}-q_{a v} \cdot p_{\mu}^{a} .
$$

The simplectic form (2.9) defines the Poisson bracket:

$$
\left\{q_{a}^{\mu}, p_{v}^{b}\right\}=\delta_{a}^{b} \cdot \eta_{\nu}^{\mu}, \quad\left\{q_{a}^{\mu}, q_{b}^{v}\right\}=\left\{p_{\mu}^{a}, p_{v}^{b}\right\}=0,
$$

in terms of which the Hamilton equations read

$\frac{\partial q_{a}^{\mu}}{\partial \tau_{b}}=\left\{H_{b}, q_{a}^{\mu}\right\}, \quad \frac{\partial p_{\mu}^{a}}{\partial \tau_{b}}=\left\{H_{b}, p_{\mu}^{a}\right\}, \quad a, b=1,2, \ldots, N,(2.12)$ and their integrability conditions:

$$
\left\{H_{a}, H_{a^{\prime}}\right\}=0, \quad \forall a^{\prime} \neq a .
$$

Assuming that the conditions (2.13) are fulfilled by the Hamiltonians, the Hamilton equations (2.12) are integrable, i.e., there exist functions $f_{a}^{\mu}\left(\tau_{1}, \ldots, \tau_{N} ; q_{b}^{v}, p_{\rho}^{c}\right)$ and $g_{\mu}^{a}\left(\tau_{1}, \ldots, \tau_{N} ; q_{b}^{v}, p_{\rho}^{c}\right)$ which are solutions of $(2.12)$ and

$$
f_{a}^{\mu}\left(0, \ldots, 0 ; q_{b}^{v}, p_{\rho}^{c}\right)=q_{a}^{\mu}, \quad g_{\mu}^{a}\left(0, \ldots, 0 ; q_{b}^{v}, p_{\rho}^{c}\right)=p_{\mu}^{a} .
$$

However, there is still one important difference between these solutions and the integral (2.4). In general, $f_{a}^{\mu}$ and $g_{\mu}^{a}$ do not accomplish the individuality condition and depend essentially on more than one of the parameters $\left(\tau_{1}, \ldots, \tau_{N}\right)$. Furthermore, if we require $f_{a}^{\mu}$ and $g_{\mu}^{a}$ to depend only on $\tau_{a}(\forall a=1, \ldots, N)$, then it turns out-no-interaction theorem ${ }^{3}$ - that only free particle Hamiltonian systems are allowed

It is for this reason that the a priori canonical coordinates $(q, p)$, have no physical meaning; therefore, we cannot identify $q_{a}^{\mu}$ with the physical position $x_{a}^{\mu}$ and, henceforth $\Omega \neq d x_{a}^{\mu} \wedge d p_{\mu}^{a}$.

In order to give some physical content to these "a priori Hamiltonian predictive systems," we must introduce the physical positions $x_{\alpha}^{\mu}(q, p)$, which we define by the individuality condition:

$$
\left\{H_{a^{\prime}}, x_{a}^{\mu}\right\}=0, \quad \forall a^{\prime} \neq a,
$$


which are $N-1$ partial differential equations on $x_{a}^{\mu}$.

A system of $N$ scalar functions $H_{a}(q, p)$ fulfilling the integrability conditions (2.13) and the individuality condition (2.14) will be called hereafter an "a priori Hamiltonian predictive system."

As far as we have assumed that (2.13) is fulfilled, the partial differential system (2.14) is integrable. Therefore, a good set of Cauchy data determines a unique solution of (2.14): $x_{a}^{\mu}(q, p)$.

We can then define the velocities

$$
\dot{x}_{a}^{\mu}(q, p) \equiv\left\{H_{a}, x_{a}^{\mu}\right\} .
$$

From (2.13) and the Jacobi identity for Poisson brackets it is obvious that

$$
\left\{H_{a^{\prime}}, \dot{x}_{a}^{\mu}\right\}=0, \quad \forall a^{\prime} \neq a .
$$

If the Jacobian $\left|\partial\left(x_{a}^{\mu}, \dot{x}_{b}^{\nu}\right) / \partial\left(q_{a}^{\mu}, p_{v}^{b}\right)\right|$ does not vanish, the functions $x_{a}^{\mu}(q, p)$ and $\dot{x}_{b}^{\nu}(q, p)$ define a change of variables. Then, the vector field $\mathbf{H}_{a}^{*}$ associated with $H_{a}(q, p)$ by $\Omega$ can be written as

$$
\mathbf{H}_{a}^{*}=\dot{x}_{a}^{\mu} \frac{\partial}{\partial x^{a \mu}}+\xi_{a}^{\mu}(x, \dot{x}) \cdot \frac{\partial}{\partial \dot{x}^{a \mu}}, \quad a=1, \ldots, N,
$$

where $\xi_{a}^{\mu}(x(q, p), \dot{x}(q, p)) \equiv\left\{H_{a}(q, p), \dot{x}_{a}(q, p)\right\}$.

These systems are of the same type as (2.2) and, as a consequence of $(2.13)$, they obviously satisfy the conditions $(2.3)^{9}$ :

$$
\left[\mathbf{H}_{a}^{*}, \mathbf{H}_{a^{\prime}}^{*}\right]=0 .
$$

Since $H_{a}(q, p)$ are scalar functions, $\dot{x}_{a}^{\mu}$ and $\xi_{a}^{\mu}(x, \dot{x})$ are translation-invariant 4-vectors.

What has been achieved is that any "a priori Hamiltonian predictive system" (HPS) with a good set of Cauchy data for (2.14) determines a unique PIAS, provided that the positions and velocities $\left(x_{a}^{\mu}, \dot{x}_{b}^{\nu}\right)$ are a complete set of coordinates on phase space.

We have to realize that the PIAS associated with the HPS depends crucially on the Cauchy data for the position equations. In general, two different sets of Cauchy data for (2.14) associate to the same HPS two different PIAS.

In general, the PIAS (2.17) is not affine parametrized, i.e., it is not a PIPS, because the condition (2.8)

$\dot{x}_{a \mu} \cdot \xi_{a}^{\mu}(x, \dot{x})=0$ need not be fulfilled.

One question now arises: Is it possible to obtain a PIPS equivalent to $(2.17)$ via a reparametrization?

\section{REPARAMETRIZATION OF A PIAS}

Let us start from a PIAS:

$$
\mathbf{H}_{a}^{*}=\dot{x}_{a}^{\mu} \cdot \frac{\partial}{\partial x^{a \mu}}+\xi_{a}^{\mu}(x, \dot{x}) \cdot \frac{\partial}{\partial \dot{x}^{a \mu}}, \quad a=1, \ldots, N .
$$

We introduce now the following change of variables in $T M^{4 N}:\left(x_{b}, \dot{x}_{c}\right) \rightarrow\left(x_{b}, \pi_{c}\right)$ defined by

$$
\pi_{a}^{\prime \prime} \equiv \beta_{a}\left(x_{b}, \dot{x}_{c}\right) \cdot \dot{x}_{a}^{\mu},
$$

where $\beta_{a}\left(x_{b}, \dot{x}_{c}\right)$ are $N$ given scalar functions.

This transformation leaves the position invariant and only modifies the velocities' moduli. The inverse transformation is given by

$$
\dot{x}_{a}^{v}=\alpha_{a}\left(x_{b}, \pi_{c}\right) \cdot \pi_{a}^{v},
$$

where the $N$ functions $\alpha_{a}\left(x_{b}, \pi_{c}\right)$ can be obtained from

$$
\alpha_{a} \cdot \beta_{a}\left(x_{b}, \alpha_{c} \pi_{c}\right)=1, \quad a=1, \ldots, N,
$$

provided that the conditions of the implicit function theorem ${ }^{12}$ are fulfilled. Moreover, it is obvious that this occurs if and only if $\left\{\beta_{a}\left(x_{b}, \dot{x}_{c}\right) \sqrt{-\dot{x}_{a}^{2}}\right\}_{a=1, \ldots, N}$ are functionally independent.

We then define the following second-order differential systems:

$$
\mathbf{H}_{a}=\pi_{a}^{\mu} \cdot \frac{\partial}{\partial x^{a \mu}}+\theta_{a}^{\mu}(x, \pi) \cdot \frac{\partial}{\partial \pi^{a \mu}}, \quad a=1, \ldots, N,
$$

with

$$
\theta_{a}^{\mu}(x, \pi) \equiv\left(\mathbf{H}_{a}^{*} \beta_{a}\right)(x, \alpha \pi) \cdot \pi_{a}^{\mu}+\beta_{a}^{2}(x, \alpha \pi) \cdot \xi_{a}^{\mu}(x, \alpha \pi) .
$$

The following two theorems are referred to the relations between PIAS and PIPS defined by reparametrizations.

Theorem 3.1: If the functions $\beta_{a}(x, \dot{x})$ introduced in (3.1) satisfy

$$
\mathbf{H}_{a^{\prime}}^{*} \beta_{a}=0, \quad \forall a^{\prime} \neq a,
$$

then:

(i) The family of second-order differential systems $\left\{\mathbf{H}_{a}\right\}_{a=1, \ldots, N}$ defined in (3.4) is a PIAS.

(ii) Any integral of $\left\{\mathbf{H}_{a}^{*}\right\}_{a=1, \ldots, N}$ can be reparametrized into an integral of $\left\{\mathbf{H}_{a}\right\}_{a=1, \ldots, N}$.

Theorem 3.2:

Let

$\left\{\mathbf{H}_{a}^{*}=\dot{x}_{a}^{\mu} \cdot \partial / \partial x^{a \mu}+\xi_{a}^{\mu}(x, \dot{x}) \cdot \partial / \partial \dot{x}^{a \mu} ; a=1, \ldots, N\right\}$ be a

PIAS and

$\left\{\mathbf{H}_{a}=\pi_{a}^{\mu} \cdot \partial / \partial x^{a \mu}+\theta_{a}^{\mu}(x, \pi) \cdot \partial / \partial \pi^{a \mu} ; a=1, \ldots, N\right\}$ be a PIPS.

Assume that any integral $\varphi_{a}^{\mu}\left(\sigma_{a}, x, \pi\right)$ of the second can be reparametrized into an integral $\psi_{a}^{\mu}\left(\tau_{a}, x, \dot{x}\right)$ of the first. In other words, there exist $2 N$ functions $f_{a}\left(\tau_{b}, x, \dot{x}\right)$ and $\beta_{b}(x, \dot{x})$ as much differenciable as necessary, such that

$\psi_{a}^{\mu}\left(\tau_{a}, x_{b}, \dot{x}_{c}\right)=\varphi_{a}^{\mu}\left(f_{a}\left(\tau_{a}, x, \dot{x}\right), x_{b}^{\nu}, \beta_{c}(x, \dot{x}) \cdot \dot{x}_{c}^{\rho}\right)$.

Then:

(i) $f_{a}(0, x, \dot{x})=0, \quad \beta_{a}(x, \dot{x})=\left(\frac{\partial f_{a}}{\partial \tau_{a}}\right)_{(0, x, \dot{x})}^{-1}$;

(ii) $\left(\frac{\partial f_{a}}{\partial \tau_{a}}\right)_{\left(\tau_{a}, x, \dot{x}\right)}^{-1}=\beta_{a}(\psi(\tau, x, \dot{x}), \dot{\psi}(\tau, x, \dot{x}))$;

(iii) $\mathbf{H}_{a}^{*} \cdot \beta_{a}=0, \quad \forall a^{\prime} \neq a$;

(iv) $\xi_{a}^{\mu}(x, \dot{x})=\beta_{a}^{-2}(x, \dot{x}) \cdot \theta_{a}^{\mu}(x, \beta \dot{x})$

$$
+\left(\frac{\partial^{2} f_{a}}{\partial \tau_{a}^{2}}\right)_{(0, x, \dot{x})} \cdot \beta_{a} \cdot \dot{x}_{a}^{\mu} .
$$

We have to realize that the reparametrization functions $f_{a}$ depend not only on $\tau_{a}$ but also on $x_{b}^{\mu}$ and $\dot{x}_{c}^{v}$. This is due to the fact that each integral of the PIAS must be reparametrized independently of the others. This makes the problem much more involved than it seems at first sight. The foregoing two theorems establish which conditions must be imposed on the functions $f_{a}\left(\tau_{a}, x_{b}^{\mu}, \dot{x}_{c}^{v}\right)$ in order to preserve the predictive abelian character of the system after reparametri- 
zation. This complication has not been noticed in the least in previous works. ${ }^{6}$ It turns out, however, that all reparametrizations of PIAS existing in the literature happen to fulfill Eqs. (3.5) and hence keep the abelian character of the system.

\section{A NATURAL RESTRICTION ON THE CAUCHY DATA}

We have concluded in Sec. II that the PIAS associated with a given HPS depends very strongly on the Cauchy data taken to solve the position equations (2.14).

The big variety of Cauchy data for Eq. (2.14) lies in two facts:

(i) The Cauchy surface $\Sigma$ is any $(7 N+1)$ submanifold of the phase space, with the only restrictions of being Poincare invariant and of not being a characteristic of (2.14). (ii) The prescription

$$
\left[x_{a}^{\mu}-f_{a}^{\mu}(q, p)\right]_{\Sigma}=0
$$

is only restricted by good behavior under the Poincaré group.

In order to reduce somehow the wide variety of solutions of the position equations, we can furthermore introduce the orthogonality conditions (2.8), which in Hamiltonian formalism are written as

$$
\left\{H_{a}, x_{a \mu}\right\} \cdot\left\{H_{a},\left\{H_{a}, x_{a}^{\mu}\right\}\right\}=0 .
$$

A first integration of this equation can be worked out immediately and leads to

$$
\left\{H_{a}, x_{a \mu}\right\} \cdot\left\{H_{a}, x_{a}^{\mu}\right\}=-m_{a}^{2}(q, p),
$$

where $m_{a}^{2}(q, p)$ is a positive integral of motion which is usually taken to be $2 H_{a}(q, p){ }^{6}$ We shall also do it hereafter.

Although (4.3) is apparently a new differential equation to add to Eq. (2.14), we shall see in the following that it can be reduced to a condition on the Cauchy prescription (4.1).

Let us first consider a Cauchy surface satisfying the above conditions (i). It will be defined by $N-1$ scalar functions:

$$
\phi_{B}(q, p)=0, \quad B=2, \ldots, N,
$$

and no linear combination of the vectors $\mathbf{H}_{b}^{*}, b \neq a$, can be tangent to $\Sigma$. That is to say:

$$
\begin{gathered}
{\left[\sum_{c \neq a} b^{c}(q, p) \cdot\left\{H_{c}, \phi_{B}\right\}\right]_{\Sigma}=0, \quad \forall B=2, \ldots, N} \\
\Rightarrow\left[b^{c}(q, p)\right]_{\Sigma}=0, \quad \forall c \neq a,
\end{gathered}
$$

which is satisfied if and only if

$$
\operatorname{det}\left(\left\{\phi_{B}, H_{c}\right\}_{\mid \Sigma}\right) \neq 0, \quad B=2, \ldots, N, \quad c \neq a .
$$

If we want $\Sigma$ to be a good Cauchy surface for the position equations of any particle, Eq. (4.4) need to be true for any $a=1, \ldots, N$.

Let us now consider a vector field of the form

$$
\mathbf{D}_{a}=\mathbf{H}_{a}^{*}+\sum_{c \neq a} \psi_{(a)}^{c} \mathbf{H}_{c}^{*}
$$

The functions $\psi_{(a)}^{c}$ can always be chosen in such a way that $\mathbf{D}_{a \mid \Sigma}$ is tangent to $\Sigma$, that is to say,

$$
\left[\mathbf{D}_{a} \phi_{B}\right]_{\Sigma}=0, \quad \forall B=2, \ldots, N,
$$

which can also be written as

$$
\left[\left\{\phi_{B}, H_{a}\right\}+\sum_{c \neq a} \psi_{(a)}^{c}\left\{\phi_{B}, H_{c}\right\}\right]_{\Sigma}=0 .
$$

But from (4.4) the linear system (4.7) is Cramer's and the restriction to $\Sigma$ of $\psi_{(a)}^{c}(q, p)$ is uniquely determined. Substituting then these suitable values of $\psi_{(a)}^{c}$ into (4.5), we have for any $g(q, p)$

$$
\left[\mathbf{D}_{a} g\right]_{\Sigma}=\mathbf{D}_{a \mid \Sigma}\left(g_{\mid \Sigma}\right)
$$

Therefore, taking (2.14) and (4.1) into account and using (4.8), we can write

$$
\left[\mathbf{H}_{a}^{*} x_{a}^{\mu}\right]_{\Sigma}=\left[\mathbf{D}^{a} x_{a}^{\mu}\right]_{\Sigma}=\mathbf{D}_{a \mid \Sigma} f_{a}^{\mu} .
$$

Theorem 4.1: The solution of Eq. (2.14) with the set of Cauchy data $\left[x_{a}^{\mu}-f_{a}^{\mu}(q, p)\right]_{\Sigma}=0$ satisfy also the condition (4.3) if and only if

$$
\left(\mathbf{D}_{a \mid \Sigma} f_{a \mu}\right)\left(\mathbf{D}_{a \mid \Sigma} f_{a}^{\mu}\right)=-m_{a}^{2}(q, p) \text {. }
$$

Proof: (a) (if) Let $x_{a}^{\mu}(q, p)$ be the solution of $(2.14)$ with these Cauchy data, and let us define $g_{a}(q, p) \equiv\left\{H_{a}, x_{a}^{\mu}\right\}$

- $\left\{H_{a}, x_{a \mu}\right\}$. From (4.9) and (4.10) it follows that

$$
g_{a \mid \Sigma}=-m_{a \mid \Sigma}^{2} \text {. }
$$

Then, using (2.13), (2.16), and the Jacobi identity for Poisson brackets, we have

$$
\left\{H_{a^{\prime}}, g_{a}\right\}=0, \quad a^{\prime} \neq a .
$$

Therefore, $g_{a}$ is a solution of (4.12) with the Cauchy data (4.11). But the uniqueness of solutions implies

$$
g_{a}(q, p)=-m_{a}^{2}(q, p),
$$

and the first part of the proof (if) is finished.

(b) The second part of the proof (only if) is immediate.

\section{APPLICATION}

We shall here apply the results obtained in the last section to the family of models presented in Ref. 13 and given by the Hamiltonian functions:

$$
\begin{aligned}
H_{b}= & \alpha\left(P \cdot y_{b}\right)-\beta P^{2}-\gamma\left[\varepsilon^{A} y_{A}^{2}+\sum_{A<A^{\prime}}\left(y_{A} \cdot y_{A^{\prime}}\right)\right] \\
& +V\left(\tilde{y}_{B}, \tilde{z}_{C}\right)
\end{aligned}
$$

where

$$
\begin{aligned}
& P^{\mu} \equiv \varepsilon^{a} p_{a}^{\mu}, \quad y_{B}^{\mu} \equiv(1 / N) P^{\mu}-p_{B}^{\mu}, \quad z_{c}^{v} \equiv q_{c}^{v}-q_{1}^{v},(5.2 \mathrm{a}) \\
& y_{\mathrm{I}}^{\mu} \equiv-\varepsilon^{A} y_{A}^{\mu}, \\
& \tilde{a}^{\mu} \equiv \pi_{v}^{\mu} \cdot a^{v}, \quad \pi_{v}^{\mu} \equiv \eta_{v}^{\mu}-P^{\mu} P_{v} / P^{2}, \\
& \varepsilon^{A}=1, a, b, c=1,2, \ldots, N, A, B, C=2,3, \ldots, N, \text { and } \alpha, \beta, \gamma \text { are }
\end{aligned}
$$
arbitrary real constants.

In these models, the integration of the position equations (2.14) has been worked out taking the Cauchy data:

$$
\left[x_{a}^{\mu}-q_{a}^{\prime \prime}\right]_{\Sigma}=0, \quad \Sigma \equiv\left\{(q, p) \in T M^{4 N} \mid\left(P \cdot z_{A}\right)=0\right\} .
$$

However, since the 4-velocities $\left\{H_{a}, x_{a}^{\mu}\right\}$ have not constant moduli the parameters $\tau_{a}, a=1, \ldots, N$, along the world lines of the particles, are not the proper times.

We shall here relax the prescription (5.3) and take

$$
\left[x_{a}^{\mu}-\left(\tilde{q}_{a}^{\mu}+h_{a}(q, p) \cdot P^{\mu}\right)\right]_{\Sigma}=0,
$$

where $h_{a}(q, p)$ is an arbitrary scalar function which will be 
fixed by requiring (4.10).

Since we are only interested in the values of $h_{a}(q, p)$ on $\Sigma \equiv\left\{\left(P \cdot z_{B}\right)=0\right\}$, it will only depend on the variables

$P^{2}, P \cdot X, P \cdot y_{B}, \widetilde{X}^{\mu}, \tilde{z}_{B}^{\mu}, \tilde{y}_{A}^{\mu}$. Also, since $\Sigma$ is translation-invariant and $x_{a}^{\mu}$ and $q_{a}^{\mu}$ change like

$x_{a}^{\mu} \rightarrow x_{a}^{\mu}+\epsilon^{\prime \prime}, q_{a}^{\mu} \rightarrow q_{a}^{\mu}+\epsilon^{\mu}$ under a translation $\epsilon^{\prime \prime}$, we have that $h_{a}(q, p)$ must change like

$$
h_{a} \rightarrow h_{a}+\epsilon \cdot P / P^{2} \text {. }
$$

Hence, we can write

$$
h_{a}=P \cdot X / P^{2}+\kappa_{a},
$$

where $\kappa_{a}$ is translation-invariant and, therefore, it only depends on $P^{2}, P \cdot y_{B}, \tilde{z}_{C}^{u}, \tilde{y}_{B}^{v}$.

We shall here study the case $a=1$. Then we can obtain the others $x_{a}^{\mu}(q, p), a \neq 1$, by a simple permutation of indices.

The vector field $D_{1}$ introduced in (4.5) is given in this particular case by

$$
\begin{aligned}
\mathbf{D}_{1}= & \frac{1}{\alpha P^{2}+N \gamma\left(P \cdot y_{1}\right)} \\
& \times\left[\alpha P^{2} \cdot \frac{\partial}{\partial \lambda}+\sum_{B=2}^{N} N \gamma P\left(y_{1}-y_{B}\right) \cdot \frac{\partial}{\partial \lambda_{B}}\right],
\end{aligned}
$$

where use has been made of the new parameters $\left\{\lambda, \lambda_{B}, B=2, \ldots, N\right\}$, which are related to the $\left\{\tau_{a}\right\}_{a=1, \ldots, N}$, by $^{13}$

$$
\lambda=(1 / N) \varepsilon^{a} \tau_{a}, \quad \lambda_{B}=\tau_{1}-\tau_{B}
$$

and

$$
\begin{aligned}
& \frac{\partial}{\partial \lambda}=\varepsilon^{a} \frac{\partial}{\partial \tau_{a}}=\varepsilon^{a}\left\{H_{a},-,\right\} \\
& \frac{\partial}{\partial \lambda_{B}}=\frac{1}{N} \frac{\partial}{\partial \lambda}-\frac{\partial}{\partial \tau_{B}}=-\alpha\left\{\left(P \cdot y_{B}\right),-\right\}
\end{aligned}
$$

Then, the condition (4.10) on the Cauchy data (5.4) implies that, on $\Sigma$,

$$
\dot{h}_{1}=\left[\left(1 /-P^{2}\right) \cdot\left(m_{1}^{2}+\dot{\tilde{q}}_{1}^{2}\right)\right]^{1 / 2},
$$

where the dot means derivation by $\mathbf{D}_{1}$. The sign + have been chosen in order to ensure the future orientation of $\dot{x}_{1}^{\mu}$.

Working out the necessary derivatives, we obtain

$$
\begin{aligned}
\dot{\tilde{q}}_{1}^{\mu}= & -\frac{N \cdot \gamma \cdot \alpha \cdot P^{2}}{\alpha \cdot P^{2}+N \cdot \gamma \cdot\left(P y_{1}\right)} \\
& \times\left[\tilde{y}_{1}^{\mu}+\left(P^{2}\right)^{-1} \cdot \varepsilon^{\alpha}\left(P \cdot y_{\alpha}\right) \cdot \tilde{y}_{a}^{\mu}\right],
\end{aligned}
$$

where, as was pointed out in the detailed study of these models, ${ }^{13} P^{2}$ and $\left(P \cdot y_{B}\right)$ are integrals of motion and the $\tilde{y}_{a}^{\mu}$ only depend on $\lambda$. Therefore, the right-hand side of (5.9) can be expressed as

$$
\tilde{\psi}^{\mu}=\alpha^{A} \cdot \tilde{y}_{\boldsymbol{A}}^{\mu},
$$

where $\alpha^{A}\left(P^{2}, P y_{B}\right)$ are integrals of motion.

The evolution of $\tilde{y}_{A}^{\mu}$ is described by ${ }^{13}$

$$
\begin{aligned}
& \frac{d \tilde{y}_{A}^{\mu}}{d \lambda}=N \cdot\left\{V, \tilde{y}_{A}^{\mu}\right\}, \\
& \frac{d \tilde{z}_{A}^{\mu}}{d \lambda}=N \cdot\left\{V, \tilde{z}_{A}^{\mu}\right\}+N \cdot \gamma \cdot\left(\tilde{y}_{A}^{\mu}+\varepsilon^{B} \tilde{y}_{B}^{\mu}\right) .
\end{aligned}
$$

Finally, substituting the solution of $(5.10)$ in (5.9) and introducing it in (5.8), we obtain $h_{1}(q, p)_{\mid \Sigma}$ by a simple integration.

In the following we shall study in detail the particular case of an oscillatorlike potential:

$$
\begin{aligned}
V & =-\frac{1}{2} k \sum_{a<a^{\prime}}\left(\tilde{q}_{a}-\tilde{q}_{a^{\prime}}\right)^{2} \\
& =-\frac{1}{2} k\left[(N-1) \cdot \epsilon^{A} \tilde{z}_{A}^{2}-2 \cdot \sum_{B<B^{\prime}}\left(\tilde{z}_{B} \cdot \tilde{z}_{B^{\prime}}\right)\right] .
\end{aligned}
$$

Equation (5.10) becomes

$$
\frac{d \tilde{y}_{A}^{\mu}}{d \lambda}=N^{2} k \tilde{r}_{A}^{\mu}, \quad \frac{d \tilde{r}_{A}^{\mu}}{d \lambda}=-N \cdot \gamma \cdot \tilde{y}_{A}^{\mu}
$$

with

$$
\begin{aligned}
& \tilde{r}_{A}^{\mu} \equiv \tilde{z}_{A}^{\mu}-(1 / N) \varepsilon^{B} \tilde{z}_{B}^{\mu}, \quad A, B=2, \ldots, N, \\
& \tilde{r}_{1}^{\mu} \equiv-(1 / N) \varepsilon^{B} \tilde{z}_{B}^{\mu} .
\end{aligned}
$$

If we now define $\tilde{\varphi}^{\mu} \equiv \alpha^{B} \tilde{r}_{B}^{\mu}$, we can write

$$
\frac{d \tilde{\psi}^{\mu}}{d \lambda}=N^{2} \cdot k \cdot \tilde{\varphi}^{\mu}, \quad \frac{d \tilde{\varphi}^{\mu}}{d \lambda}=-N \cdot \gamma \cdot \tilde{\psi}^{\mu},
$$

whose general solution is

$\tilde{\psi}^{\mu}(\lambda)=\widetilde{R}^{\mu} \cdot \sin \omega \lambda+\tilde{S}^{\mu} \cdot \cos \omega \lambda, \quad \omega \equiv\left(N^{3} k \gamma\right)^{1 / 2}$

$\tilde{\varphi}^{\mu}(\lambda)=\frac{\omega}{N^{2} \cdot k}\left[\widetilde{R}^{\mu} \cos \omega \lambda-\tilde{S}^{\mu} \sin \omega \lambda\right]$,

where $\widetilde{R}^{\mu}$ and $\tilde{S}^{\mu}$ depend on the initial conditions.

Substituting (5.15) into (5.8) and (5.9), it yields

$$
\begin{aligned}
\dot{h}_{\mathrm{I}}= & \left(1 / \sqrt{-P^{2}}\right)\left[m_{1}^{2}+\widetilde{R}^{2} \cdot \sin ^{2} \omega \lambda+\tilde{S}^{2} \cdot \cos ^{2} \omega \lambda\right. \\
& +2(\widetilde{R} \cdot \tilde{S}) \cos \omega \lambda \cdot \sin \omega \lambda] .
\end{aligned}
$$

Since $P^{2}$ and $\left(P \cdot y_{B}\right)$ do not depend either on $\lambda$ nor on $\lambda_{B}$ and $\tilde{y}_{A}^{\mu}$ only depends on $\lambda$, we can write, taking $\left(5.9^{\prime}\right)$ into account,

$$
\tilde{\psi}^{\mu}(\lambda)=\bar{\psi}^{\mu}(b \sigma)
$$

where $b$ is a constant of motion which we derive in Appendix $A$ and $\sigma$ is the parameter along the integral curves of $\mathbf{D}_{1}$ :

$$
\mathbf{D}_{1}=\frac{d}{d \sigma} \text {. }
$$

Hence, introducing this in $(5.16)$ yields

$$
\begin{aligned}
\frac{d h_{1}}{d \sigma}= & \frac{1}{\sqrt{-P^{2}}} \cdot\left[m_{1}^{2}+\widetilde{R}^{2} \cdot \sin ^{2}(\omega b \sigma)+\tilde{S}^{2}\right. \\
& \left.\cdot \cos ^{2}(\omega b \sigma)+2(\widetilde{R} \cdot \tilde{S}) \sin (\omega b \sigma) \cdot \cos (\omega b \sigma)\right],
\end{aligned}
$$

which, after some manipulations, leads to

$$
\frac{d h_{1}}{d \sigma}=\sqrt{A^{2}-B^{2} \sin ^{2}(\omega b \sigma-\theta)},
$$

with

$$
\begin{aligned}
& A^{2}=\left(1 /-P^{2}\right)\left[m_{1}^{2}+\frac{1}{2}\left(\widetilde{R}^{2}+\tilde{S}^{2}\right)\right]+\frac{1}{2} B^{2} \geqslant 0, \\
& B^{2}=\frac{2}{-P^{2}} \sqrt{(\widetilde{R} \tilde{S})^{2}+\frac{1}{4}\left(\widetilde{R}^{2}-\tilde{S}^{2}\right) \geqslant 0} \\
& \tan 2 \theta=2(\tilde{R} \cdot \tilde{S}) /\left(\tilde{S}^{2}-\widetilde{R}^{2}\right) .
\end{aligned}
$$

Since $B^{2} \leqslant A^{2}$, the solution of (5.19) can be expressed in 
terms of an elliptic integral of the first kind ${ }^{14}$ :

$$
h_{1}(\sigma)=\frac{A}{\omega b} \cdot \mathrm{E}\left(\frac{B}{A}, \omega b \sigma-\theta\right)+\Gamma,
$$

where $\Gamma$ satisfies $\mathbf{D}_{1} \Gamma=0$.

We now have to explain what does this result mean. We are looking for a function $h_{1}\left(P^{2}, P X, P y_{B}, \widetilde{X}^{\mu}, \tilde{y}_{B}^{v}, \tilde{z}_{c}^{\rho}\right)$ on $\Sigma$ satisfying the ordinary differential equation (5.19). A unique solution of it is determined giving a suitable set of initial conditions, i.e., a $7 N$-submanifold $\Sigma^{*}$ of $\Sigma$ such that it is not a characteristic of $\mathbf{D}_{1}$, and the prescribed value of $h_{1}$ on $\Sigma^{*}$. The situation is visualized in Fig. 1.

Equation (5.21) gives us $h_{1}(Q)$ in terms of $Q_{0}$ and $\sigma$ [as is obvious $A^{2}, B^{2}, \theta$ are given in terms of the variables in $Q_{0}$, the initial point for the trajectory of (5.14)]. However, we want our final result in terms of the coordinates of $Q$.

In Appendix $\mathrm{B}$ the expressions of $A^{2}$ and $B^{2}$ in terms of constants of motion are given (B4). We also give there an expression (B7) for the phase $\theta$ in terms of $Q$. Substituting (B7) into (5.21), it yields

$$
h_{1}(Q)=\frac{A}{\omega b} \mathrm{E}\left(\frac{B}{A},-\rho\right)+\Gamma,
$$

with

$$
\rho \equiv \frac{1}{2} \arctan \frac{2 \sqrt{N \gamma}(\tilde{\varphi} \cdot \tilde{\psi})}{\tilde{\psi}^{2}-N \gamma \tilde{\varphi}^{2}}
$$

where

$$
\begin{aligned}
& \tilde{\varphi}^{\mu}=-\frac{N \gamma \alpha P^{2}}{\alpha P^{2}+N \gamma\left(P \cdot y_{1}\right)}\left[\tilde{r}_{1}^{\mu}+\frac{1}{P^{2}} \varepsilon^{a}\left(P y_{a}\right) \tilde{r}_{a}^{\mu}\right], \\
& \tilde{\psi}^{\mu}=-\frac{N \gamma \alpha P^{2}}{\alpha P^{2}+N \gamma\left(P y_{1}\right)}\left[\tilde{y}_{1}^{\mu}+\frac{1}{P^{2}} \varepsilon^{a}\left(P y_{a}\right) \cdot \tilde{y}_{a}^{\mu}\right] .
\end{aligned}
$$

The integral of motion $\Gamma$ is determined by fixing the initial conditions. If, for instance, we take

$$
\Sigma * \equiv\{P \cdot X=0\}, \quad h_{1 \mid \Sigma^{*}}=0,
$$

we have, taking (5.22) into account, that

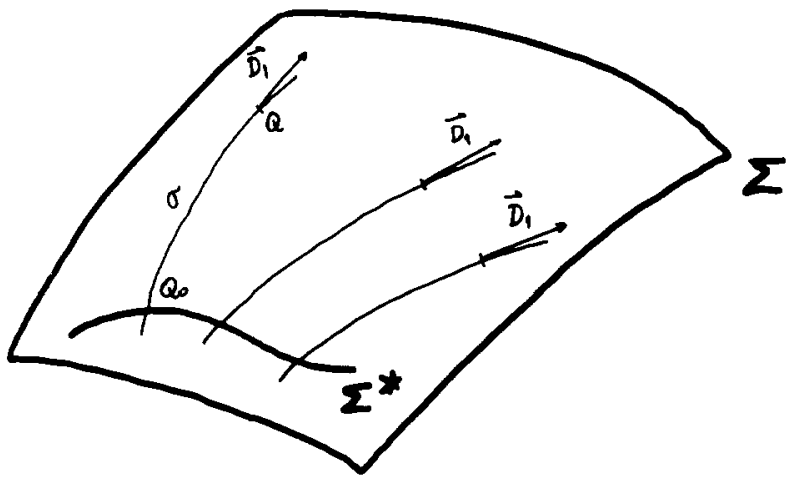

$$
\begin{aligned}
& Q \equiv\left(P^{2}, P_{y_{B}} ; P X, \tilde{z}^{\mu}, \tilde{y}_{B}^{p}, \tilde{z}_{c}^{\nu}\right) \\
& Q_{0} \equiv\left(P^{2}, P_{y_{B}} ; P Z_{0}, \tilde{z}_{0}^{\mu}, \tilde{y}_{\theta}^{\rho}, \tilde{z}_{0}^{\nu}\right)
\end{aligned}
$$

FIG. 1. $\sigma=$ variation of the parameter between $Q_{0}$ and $Q$.

$$
\Gamma=-\frac{A}{\omega b} \cdot \mathrm{E}\left(\frac{B}{A},-\rho-\omega \lambda\right)
$$

and

$$
h_{1}(Q)=\frac{A}{\omega b}\left[\mathrm{E}\left(\frac{B}{A},-\rho\right)-\mathrm{E}\left(\frac{B}{A},-\rho-\omega \lambda\right)\right],
$$

where $\lambda$ is obtained from $(\mathrm{A} 2)$ :

$$
\lambda=\left[2 N \beta P^{2}-\left(1 / P^{2}\right) N \gamma \varepsilon^{a}\left(P y_{a}\right)^{2}\right]^{-1} \cdot(P \cdot X) .
$$

\section{CONCLUSIONS}

This paper is a study of the equivalence between two different versions of predictive relativistic mechanics.

An $N$-particle system is described in the " $a$ priori" $\mathrm{Ha}$ miltonian formalism ${ }^{6}$ by $N$ Poincaré-invariant functions $H_{a}$ such that their mutual Poisson brackets vanish. The physical position are related to the canonical variables through the individuality conditions (2.14), which admit a wide class of solutions depending on the prescribed Cauchy data. This is the worst feature of this formalism because fixing only the Hamiltonian has not any physical meaning.

Hence, in this framework the physical model must be established in two steps: (i) the Hamiltonian functions and (ii) the Cauchy data for the position equations. Then, integrating these equations we can associate a Poincaré-invariant abelian system (PIAS) to the given Hamiltonian model (HPS).

In general, the PIAS obtained in this way does not fulfill the condition of constancy of the velocities' moduli, and therefore it is not a Poincaré-invariant predictive system (PIPS). We have shown in Sec. III that by means of a reparametrization a PIPS can be associated with a given PIAS. This reparametrization is not unique. It involves a change of coordinates of the form:

$$
\left(x_{a}^{\mu}, \dot{x}_{b}^{v}\right) \rightarrow\left(x_{a}^{\mu}, \pi_{b}^{v}\right), \quad \pi_{b}^{v} \equiv \beta_{b}(x, \dot{x}) \cdot \dot{x}_{b}^{v} .
$$

We have seen in Sec. III what conditions must be fulfilled by the proportionality factors $\beta_{b}(x, \dot{x})$ in order to preserve the commutativity conditions (2.3).

If a given reparametrization transforms a PIAS into a PIPS, the new variables $\left(x_{a}^{\mu}, \pi_{b}^{\nu}\right)$ must verify:

$\pi_{a}^{\mu} \pi_{a \mu}=-m_{a}^{2}, a=1, \ldots, N$, where $\left\{m_{a}^{2}, a=1 \cdots N\right\}$ are $N$ arbitrary functionally independent constants of motion.

Therefore, a given PIAS is reparametrized into a PIPS by as much reparametrizations as possible choices of the set $\left.m_{a}^{2}, a=1 \ldots N\right\}$. The most natural choice of these constants are the $N$ Hamiltonians.

However, the Lie derivatives of the symplectic form $\Omega$ with respect to the proper time along the individual world lines (i.e., the derivatives $\mathscr{L}\left(\mathbf{H}_{a}\right) \Omega$ being $\mathbf{H}_{a}$, the tangent vector fields of the PIPS, $a=1 \cdots N)$ do not vanish and hence the PIPS is not Hamiltonian, with respect to the symplectic form $\Omega$.

In Sec. IV we have considered the special situation in which the Cauchy data for the position equations lead directly to a PIPS and no reparametrization is necessary. In this case the PIPS is Hamiltonian. In Sec. V we have applied these results to the family of models presented in Ref. 6. 


\section{APPENDIX A}

The evolution of the functions $(P X),\left(P z_{A}\right), A=2, \ldots, N$ is given by ${ }^{13}$

$(P X)=(P X)_{0}+2 N \cdot \beta \cdot P^{2} \lambda+\alpha \varepsilon^{A}\left(P y_{A}\right) \cdot \lambda_{A}$,

$\left(P z_{A}\right)=\left(P z_{A}\right)_{0}+N \cdot \gamma\left[\left(P y_{A}\right)+\varepsilon^{B}\left(P y_{B}\right)\right] \cdot \lambda+\alpha P^{2} \lambda_{B}$.

If we constrain the evolution to remain on

$\Sigma \equiv\left\{P z_{A}=0, A=2, \ldots, N\right\}$, we have

$\lambda_{B}=-\frac{N \gamma}{\alpha P^{2}}\left[\left(P y_{B}\right)+\varepsilon^{A}\left(P y_{A}\right)\right] \cdot \lambda$,

$(P X)_{\Sigma}=(P X)_{0}+\lambda \cdot\left[2 N \cdot \beta \cdot P^{2}-\frac{1}{P^{2}} \cdot N \cdot \gamma \cdot \varepsilon^{a}\left(P y_{a}\right)^{2}\right]$.

Therefore, we can write

$\lambda=\frac{(P X)_{\Sigma}-(P X)_{0}}{2 N \cdot \beta \cdot P^{2}-\left(1 / P^{2}\right) \cdot N \cdot \gamma \cdot \varepsilon^{a}\left(P y_{a}\right)^{2}}$.

We can also relate the parameters $\lambda$ and $\sigma$

$$
\begin{aligned}
\frac{d(P X)_{\Sigma}}{d \lambda}= & 2 N \beta P^{2}-\frac{1}{P^{2}} \cdot N \cdot \gamma \cdot \varepsilon^{a}\left(P y_{a}\right)^{2}, \\
\frac{d(P X)_{\Sigma}}{d \sigma}= & \mathbf{D}_{1}(P X)_{\Sigma}=\frac{\alpha P^{2}}{\alpha P^{2}+N \cdot \gamma \cdot\left(P y_{1}\right)} \\
& \times\left[2 N \beta P^{2}-\frac{1}{P^{2}} N \cdot \gamma \cdot \varepsilon^{a}\left(P y_{a}\right)^{2}\right] ;
\end{aligned}
$$

hence

$$
b=\frac{d \lambda}{d \sigma}=\frac{\alpha P^{2}}{\alpha P^{2}+N \cdot \gamma \cdot\left(P y_{1}\right)} .
$$

\section{APPENDIX B}

We shall here derive the expressions of $A^{2}, B^{2}, \theta$ in terms of integrals of motion.

As is well known, ${ }^{15}$ the system (5.14) has the tensor integral of motion:

$$
\widetilde{H}^{\mu v} \equiv N^{2} \cdot k \cdot \tilde{\varphi}^{\mu} \cdot \tilde{\varphi}^{v}+N \cdot \gamma \cdot \tilde{\psi}^{\mu} \cdot \tilde{\psi}^{v} .
$$

Substituting (5.15) into (B1), it yields

$$
\widetilde{H}^{\mu \nu}=N \cdot \gamma \cdot\left(\widetilde{R}^{\mu} \cdot \widetilde{R}^{v}+\tilde{S}^{\mu} \cdot \tilde{S}^{v}\right) .
$$

We then define

$$
\widetilde{M}^{\mu v} \equiv \frac{1}{N \gamma} \widetilde{H}^{\mu v}=\frac{N \cdot k}{\gamma} \tilde{\varphi}^{\mu} \cdot \tilde{\varphi}^{v}+\tilde{\psi}^{\mu} \cdot \tilde{\psi}^{v},
$$

which is also a tensor integral of motion.

Using (B3), we can write

$$
\begin{aligned}
B^{2} & =\left[2 /\left(-P^{2}\right)\right] \cdot\left[-\frac{1}{4}\left(\widetilde{\boldsymbol{M}}_{\mu}^{\mu}\right)^{2}+\frac{1}{2} \widetilde{\boldsymbol{M}}^{\mu \nu} \widetilde{\boldsymbol{M}}_{\mu \nu}\right]^{1 / 2} \\
& =\left[2 /\left(-P^{2}\right)\right]\left\{\frac{1}{4}\left[(N k / \gamma) \tilde{\varphi}^{2}-\tilde{\psi}^{2}\right]^{2}+(N k / \gamma)(\tilde{\varphi} \tilde{\psi})^{2}\right\}^{1 / 2},
\end{aligned}
$$

$A^{2}=\left[1 /\left(-P^{2}\right)\right]\left(m_{1}^{2}+\frac{1}{2} \widetilde{M}_{\mu}^{\mu}\right)+\frac{1}{2} B^{2}$

$$
=\left[1 /\left(-P^{2}\right)\right]\left[m_{1}^{2}+(N k / 2 \gamma) \tilde{\varphi}^{2}+\frac{1}{2} \tilde{\psi}^{2}\right]+\frac{1}{2} B^{2},
$$

where $\tilde{\varphi}^{\mu}$ and $\tilde{\psi}^{v}$ are given in terms of $\tilde{y}_{A}^{\mu}$ and $\tilde{z}_{B}^{\nu}$ by (5.9), $\left(5.9^{\prime}\right)$, and $(5.13)$.

From (5.15) we have

$$
\begin{aligned}
& \widetilde{R}^{\mu}=\tilde{\psi}^{\mu} \cdot \sin \omega \lambda+\sqrt{N \gamma} \cdot \tilde{\varphi}^{\mu} \cdot \cos \omega \lambda, \\
& \tilde{S}^{\mu}=\tilde{\psi}^{\mu} \cdot \cos \omega \lambda-\sqrt{N \gamma} \cdot \tilde{\varphi}^{\mu} \cdot \sin \omega \lambda,
\end{aligned}
$$

and, substituting this in $(5.20 \mathrm{c})$, it yields

$\tan 2 \theta=\frac{\left(\tilde{\psi}^{2}-N \gamma \tilde{\varphi}^{2}\right) \cdot \sin (2 \omega \lambda)+2 \sqrt{N \gamma}(\tilde{\varphi} \cdot \tilde{\psi}) \cdot \cos (2 \omega \lambda)}{\left(\tilde{\psi}^{2}-N \gamma \tilde{\varphi}^{2}\right) \cdot \cos (2 \omega \lambda)-2 \sqrt{N \gamma}(\tilde{\varphi} \tilde{\psi}) \cdot \sin (2 \omega \lambda)}$,

or, equivalently,

$\theta=\omega \lambda+\rho, \quad \rho \equiv \frac{1}{2} \arctan \left[2 \sqrt{N \gamma}(\tilde{\varphi} \tilde{\psi}) /\left(\tilde{\psi}^{2}-N \gamma \cdot \tilde{\varphi}^{2}\right)\right]$.

'J. A. Wheeler and R. P. Feynman, Rev. Mod. Phys. 17, 157 (1945); J. A Wheeler and R. P. Feynman, Rev. Mod. Phys. 21, 425 (1949); H. Van Dam and E. P. Wigner, Phys. Rev. 192, 838 (1966).

${ }^{2}$ P. A. M. Dirac, Rev. Mod. Phys. 21, 392 (1949); B. Bakampian and L. H Thomas, Phys. Rev. 92, 1300 (1953); L. L. Foldy, Phys. Rev. 122, 275 (1961).

${ }^{3}$ D. G. Currie, T. F. Jordan, and E. C. G. Sudarshan, Rev. Mod. Phys. 35, $350(1963)$.

${ }^{4}$ D. G. Currie, Phys. Rev. 142, 817 (1966); R. N. Hill, J. Math. Phys. 8, 201 (1967); E. H. Kerner, J. Math. Phys. 9, 222 (1968); T. F. Jordan, Phys. Rev. 166, 1309 (1968); P. Droz Vincent, Phys. Scripta 2, 129 (1970); L. Bel, Ann. Inst. H. Poincaré 14, 189 (1971); R. Arens, Arch. Rat. Mech. Anal. 47, 255 (1972).

${ }^{5}$ P. Droz Vincent, Phys. Scripta 2, $129(1970)$.

${ }^{6}$ P. Droz Vincent, Rep. Math. Phys. 8, 79 (1975); P. Droz Vincent, Ann. Inst. H. Poincaré 27, 407 (1977); V. Iranzo, J. Llosa, F. Marqués, and A. Molina, Ann. Inst. H. Poincaré 35, 1 (1981).

${ }^{7}$ L. Bel and J. Martin, Ann. Inst. H. Poincaré 22, 173 (1975); L. Bel and X. Fustero, Ann. Inst. H. Poincaré A 25, 411 (1976).

${ }^{8}$ P. Droz Vincent, Phys. Scripta 2, $129(1970)$.

${ }^{9} \mathrm{C}$. Godbillon, Geometrie differentielle et mécanique analytique (Hermann, Paris, 1969).

${ }^{10}$ J. Llosa, doctoral thesis, Universitat de Barcelona, 1978.

${ }^{11} \mathrm{C}$. Bona, doctoral thesis, Universitat Autònoma de Barcelona, 1980.

${ }^{12}$ M. Spivak, Calculus on Manifolds (Benjamin, New York, 1965).

${ }^{13} \mathrm{~V}$. Iranzo, J. Llosa, F. Marqués, and A. Molina, Ann. Inst. H. Poincaré 35, 1 (1981).

${ }^{14} \mathrm{M}$. R. Spiegel, Handbook of Mathematical Formulas and Tables (McGraw-Hill, New York, 1970).

15J. P. Elliott, Proc. R. Soc. London Ser. A 245, 128 (1958); P. Droz-Vincent, Ann. Inst. H. Poincaré A 27, 407 (1977). 